\title{
Article
}

\section{Existence result for a singular semipositone dynamic system on time scales}

\author{
Arzu Denk Oguz ${ }^{1}$ and Fatma Serap Topal ${ }^{2, *}$ \\ 1 Department of Mathematics, Atilim University, Incek, Ankara-Turkey.; arzu.denk@hotmail.com \\ 2 Department of Mathematics, Ege University, Bornova, Izmir-Turkey. \\ * Correspondence: f.serap.topal@ege.edu.tr
}

Received: 25 October 2019; Accepted: 4 February 2020; Published: 24 March 2020.

\begin{abstract}
We concentrate on investigating the existence of positive solutions for the system of second order singular semipositone m-point boundary value problems in this article. We emphasize that the nonlinear term may take a negative value and be singular. By the properties of Green's function and applying fixed point theorem in cones, existence results for positive solutions are obtained. Also, we provide an example to make our results clear and easy for readers to understand the existence result.
\end{abstract}

Keywords: Positive solutions, fixed point theorem, semipositone problems, time scales.

MSC: 34B15, 39A10.

\section{Introduction}

$\mathbf{M}$ ulti-point boundary value problems for second order and higher order ordinary differential equations and systems arise from many fields in physics, biology and chemistry. These problems play very important role in both theory and applications [1-5].

Problems where the nonlinear terms have some singularities are referred to as singular problems in the literature and this type of differential systems appear in the study of gas dynamics, fluid mechanics, in the theory of boundary layer and so on. Because of its applications in physics, singular problems have extensively study in recent years, for example see [6-9].

For example, Asif and Khan [6] studied the existence of positive solution to a nonlinear singular system with four-point boundary conditions of the type

$$
\begin{aligned}
-x(t)^{\prime \prime} & =f(t, x(t), y(t)), \quad t \in(0,1), \\
-y(t)^{\prime \prime} & =g(t, x(t), y(t)), \quad t \in(0,1), \\
x(0) & =0, x(1)=\alpha y(\xi), \quad y(0)=0, \quad y(1)=\beta x(\eta) .
\end{aligned}
$$

In [7], Liu and Yan considered the following singular boundary value problem of Sturm Liouville differential system:

$$
\begin{aligned}
& \left(p(t) x(t)^{\prime}\right)^{\prime}+\lambda f(t, x(t), y(t))=0, \quad t \in(0,1), \\
& \left(p(t) y(t)^{\prime}\right)^{\prime}+\lambda g(t, x(t), y(t))=0, \quad t \in(0,1), \\
& \alpha x(0)-\beta p(0) x^{\prime}(0)=\gamma x(1)-\delta p(1) x^{\prime}(1)=0, \\
& \alpha y(0)-\beta p(0) y^{\prime}(0)=\gamma y(1)-\delta p(1) y^{\prime}(1)=0 .
\end{aligned}
$$

Although much interest has been observed in investigating the existence of positive solutions of dynamic equations on measure chains [9-13], very few research articles has been seen on the existence of positive solutions of dynamic systems on measure chains [14,15].

In [16], Prasad, Rao and Bharathi interested in the existence of positive solutions to the system of dynamic equations:

$$
(-1)^{n} u^{\triangle^{2 n}}(t)+\lambda p(t) f(v(\sigma(t)))=0, \quad t \in[a, b],
$$




$$
\begin{gathered}
(-1)^{n} v^{\Delta^{2 n}}(t)+\mu q(t) f(u(\sigma(t)))=0, \quad t \in[a, b], \\
\alpha_{i+1} u^{\triangle^{2 i}}(a)-\beta_{i+1} u^{\triangle^{2 i+1}}(a)=0, \gamma_{i+1} u^{\triangle^{2 i}}(\sigma(b))+\delta_{i+1} u^{\triangle^{2 i+1}}(\sigma(b))=0, \\
\alpha_{i+1} v^{\triangle^{2 i}}(a)-\beta_{i+1} v^{\triangle^{2 i+1}}(a)=0, \gamma_{i+1} v^{\triangle^{2 i}}(\sigma(b))+\delta_{i+1} v^{\triangle^{2 i+1}}(\sigma(b))=0 .
\end{gathered}
$$

Problems of this type where the nonlinear term may change sign are referred to as semipositone problems in the literature. Semipositone differential systems appear in the study of chemical reactors [17].

The above works motivates us to consider the nonlinear singular semipositone system of $m$-point boundary value problem (SSS) in this paper.

$$
\left\{\begin{array}{l}
-\left[p(t) u_{i}^{\triangle}(t)\right]^{\nabla}+q(t) u_{i}(t)=f_{i}\left(t, u_{1}(t), u_{2}(t)\right)+h_{i}(t), \quad t \in(a, b), \quad i=1,2, \\
\alpha u_{i}(a)-\beta u_{i}^{[\triangle]}(a)=\sum_{k=1}^{m-2} \alpha_{k} u_{i}\left(\xi_{k}\right), \quad \gamma u_{i}(b)+\delta u_{i}^{[\triangle]}(b)=\sum_{k=1}^{m-2} \beta_{k} u_{i}\left(\xi_{k}\right), \quad i=1,2,
\end{array}\right.
$$

where $\alpha, \beta, \gamma, \delta, \xi_{k}, \alpha_{k}, \beta_{k}$ (for $k \in\{1,2, \ldots, m-2\}$ ) are complex constants such that $|\alpha|+|\beta| \neq 0,|\gamma|+|\delta| \neq 0$ and $\xi_{k} \in \mathrm{T} \backslash\{a, b\}, q: \mathrm{T} \rightarrow \mathcal{C}$ is a continuous function, $p: \mathrm{T} \rightarrow \mathcal{C}$ is $\nabla$ - differentiable on $\mathrm{T}_{k}, p(t) \neq 0$ for all $t \in \mathrm{T}, p^{\nabla}: \mathrm{T}_{k} \rightarrow \mathcal{C}$ is continuous, $f_{1}$ and $f_{2}:(a, b) \times[0, \infty) \times[0, \infty) \rightarrow[0, \infty)$ are continuous and may be singular at $t=a, b$ and $h_{1}$ and $h_{2}:(a, b) \rightarrow(-\infty, \infty)$ are Lebesgue integrable and may have finitely many singularities in $[a, b]$.

By an interval $(a, b)$, we mean the intersection of the real interval $(a, b)$ with the given time scale $\mathrm{T}$. Some preliminary definitions and theorems on time scales can be found in the books $[18,19]$.

Compared to previous work in this field, this study presented three new features. Firstly, the nonlinear term is allowed to change sign and tends to negative infinity. Secondly, is allowed to have finitely many singularities in $[a, b]$. Lastly, the boundary condition taken up generalizes the conditions of many problems in the literature. By using the cone theory technique, we establish some sufficient conditions for the existence of multiple positive solutions to the SSS (1). The rest of the paper is organized as follows: Section 2 gives some inequalities for Green's function and some results which are needed later. Criteria for the existence of positive solutions of the SSS (1) is established in Section 3 and uses fixed point index theorem. In addition, an example is given to illustrate the applications of main result.

\section{The preliminary Lemmas}

We shall work in the space $E=C([a, b] ; R) \times C([a, b] ; R)$. The space $E$ is a Banach space if it is endowed with the norm as follows:

$$
\left\|\left(u_{1}, u_{2}\right)\right\|=\left\|u_{1}\right\|+\left\|u_{2}\right\|, \quad\left\|u_{i}\right\|=\max _{t \in[a, b]}\left|u_{i}(t)\right|, \quad i=1,2,
$$

for any $\left(u_{1}, u_{2}\right) \in E$. For any $u=\left(u_{1}, u_{2}\right), v=\left(v_{1}, v_{2}\right) \in E$, we denote

$$
u \leq v \Leftrightarrow u_{i}(t) \leq v_{i}(t), \quad t \in[a, b], \quad i=1,2 .
$$

In the following, let us define a cone $P$ of $E$ by

$$
P=\left\{\left(u_{1}, u_{2}\right) \in E: u_{i}(t) \geq g(t)\left\|u_{i}\right\|, \quad t \in[a, b], \quad i=1,2\right\},
$$

where $g$ is defined by

$$
g(t):=\min _{t \in[a, b]}\left\{\frac{\phi_{1}(t)}{\phi_{1}(b)}, \frac{\phi_{2}(t)}{\phi_{2}(a)}\right\},
$$

and $\phi_{1}, \phi_{2}$ are the solutions of the linear problems

$$
\begin{aligned}
& {\left[p(t) \phi_{1}^{\triangleright}(t)\right]^{\nabla}-q(t) \phi_{1}(t)=0, \quad t \in(a, b),} \\
& \phi_{1}(a)=\beta, \quad \phi_{1}^{[\triangle]}(a)=\alpha,
\end{aligned}
$$

and 


$$
\begin{aligned}
& {\left[p(t) \phi_{2}^{\triangle}(t)\right]^{\nabla}-q(t) \phi_{2}(t)=0, \quad t \in(a, b),} \\
& \phi_{2}(b)=\delta, \quad \phi_{2}^{[\triangle]}(b)=-\gamma,
\end{aligned}
$$

respectively. Let $G(t, s)$ be the Green's function for the boundary value problem

$$
\begin{aligned}
& -\left[p(t) u^{\triangle}(t)\right]^{\nabla}+q(t) u(t)=0, \quad t \in(a, b), \\
& \alpha u(a)-\beta u^{[\triangle]}(a)=0, \quad \gamma u(b)+\delta u^{[\triangle]}(b)=0,
\end{aligned}
$$

is given by

$$
G(t, s):=\frac{1}{d}\left\{\begin{array}{ll}
\phi_{1}(s) \phi_{2}(t), & a \leq s \leq t \leq b \\
\phi_{1}(t) \phi_{2}(s), & a \leq t \leq s \leq b
\end{array} .\right.
$$

where $d=-W_{t}\left(\phi_{1}, \phi_{2}\right)=p(t)\left[\phi_{1}^{\triangle}(t) \phi_{2}(t)-\phi_{1}(t) \phi_{2}^{\triangle}(t)\right]$.

Let us define

$$
\Omega:=\left|\begin{array}{cc}
-\sum_{k=1}^{m-2} \alpha_{k} \phi_{1}\left(\xi_{k}\right) & d-\sum_{k=1}^{m-2} \alpha_{k} \phi_{2}\left(\xi_{k}\right) \\
d-\sum_{k=1}^{m-2} \beta_{k} \phi_{1}\left(\xi_{k}\right) & -\sum_{k=1}^{m-2} \beta_{k} \phi_{2}\left(\xi_{k}\right)
\end{array}\right|
$$

and assume that the following conditions are satisfied:

$\left(H_{1}\right) p(t)>0, q(t) \geq 0$,

$\left(H_{2}\right) \alpha, \gamma \geq 0, \beta, \delta>0, \alpha_{k}, \beta_{k} \geq 0$ for $k \in\{1,2, \ldots, m-2\}$,

$\left(H_{3}\right)$ If $q(t) \equiv 0$, then $\alpha+\gamma>0$,

$\left(H_{4}\right) \Omega<0, d-\sum_{k=1}^{m-2} \alpha_{k} \phi_{2}\left(\xi_{k}\right)>0, d-\sum_{k=1}^{m-2} \beta_{k} \phi_{1}\left(\xi_{k}\right)>0$.

To prove the main results, we will employ following lemmas.

Lemma 1. [18] Under the conditions $\left(H_{1}\right)$ and $\left(H_{2}\right)$, the solutions $\phi_{1}(t)$ and $\phi_{2}(t)$ posses the following properties:

$$
\phi_{1}(t), \phi_{2}(t) \geq 0, \quad \phi_{1}^{[\triangle]}(t) \geq 0, \quad \phi_{2}^{[\triangle]}(t) \leq 0, \quad t \in[a, b] .
$$

Lemma 2. [18] If the conditions $\left(H_{1}\right)-\left(H_{3}\right)$ are hold, then $G(t, s)>0$ for $t, s \in[a, b]$.

Lemma 3. [20] Assume that $\left(H_{1}\right)-\left(H_{3}\right)$ hold. Then

$$
g(t) G(s, s) \leq G(t, s) \leq G(s, s), \quad t, s \in[a, b],
$$

where $g$ is given in equation (2).

We consider the following boundary value problem

$$
\begin{aligned}
& -\left[p(t) u^{\triangle}(t)\right]^{\nabla}+q(t) u(t)=y(t), \quad t \in(a, b), \\
& \alpha u(a)-\beta u^{[\triangle]}(a)=\sum_{k=1}^{m-2} \alpha_{k} u\left(\xi_{k}\right), \quad \gamma u(b)+\delta u^{[\triangle]}(b)=\sum_{k=1}^{m-2} \beta_{k} u\left(\xi_{k}\right) .
\end{aligned}
$$

Lemma 4. [12] Let the conditions $\left(H_{1}\right)-\left(H_{3}\right)$ be hold. Assume that $\Omega: \neq 0$. Then for $y \in C([a, b])$, the boundary value problem given in equations (4)-(5) has a unique solution

$$
u(t)=\int_{a}^{b} G(t, s) y(s) \nabla s+A(y) \phi_{1}(t)+B(y) \phi_{2}(t),
$$

where $G(t, s)$ is given in equation (3),

$$
A(y):=\frac{1}{\Omega}\left|\begin{array}{cc}
\sum_{k=1}^{m-2} \alpha_{k} \int_{a}^{b} G\left(\xi_{k}, s\right) y(s) \nabla s & d-\sum_{k=1}^{m-2} \alpha_{k} \phi_{2}\left(\xi_{k}\right) \\
\sum_{k=1}^{m-2} \beta_{k} \int_{a}^{b} G\left(\xi_{k}, s\right) y(s) \nabla s & -\sum_{k=1}^{m-2} \beta_{k} \phi_{2}\left(\xi_{k}\right)
\end{array}\right|,
$$




$$
B(y):=\frac{1}{\Omega}\left|\begin{array}{cc}
-\sum_{k=1}^{m-2} \alpha_{k} \phi_{1}\left(\xi_{k}\right) & \sum_{k=1}^{m-2} \alpha_{k} \int_{a}^{b} G\left(\xi_{k}, s\right) y(s) \nabla s \\
d-\sum_{k=1}^{m-2} \beta_{k} \phi_{1}\left(\xi_{k}\right) & \sum_{k=1}^{m-2} \beta_{k} \int_{a}^{b} G\left(\xi_{k}, s\right) y(s) \nabla s
\end{array}\right| .
$$

Lemma 5. [5] Let $\left(H_{1}\right)-\left(H_{4}\right)$ hold. If $y \in C([a, b],[0, \infty))$, then the solution $u$ of the boundary value problem (4)-(5) satisfies $u(t) \geq 0$, for $t \in[a, b]$.

Lemma 6. If $\int_{a}^{b} G(s, s) y(s) \nabla s<\infty$, then the following inequalities are satisfied:

$$
A(y) \leq A \int_{a}^{b} G(s, s) y(s) \nabla s, \quad B(y) \leq B \int_{a}^{b} G(s, s) y(s) \nabla s,
$$

where

$$
\begin{aligned}
& A=\frac{1}{\Omega}\left|\begin{array}{cc}
\sum_{k=1}^{m-2} \alpha_{k} & d-\sum_{k=1}^{m-2} \alpha_{k} \phi_{2}\left(\xi_{k}\right) \\
\sum_{k=1}^{m-2} \beta_{k} & -\sum_{k=1}^{m-2} \beta_{k} \phi_{2}\left(\xi_{k}\right)
\end{array}\right|, \\
& B=\frac{1}{\Omega}\left|\begin{array}{cc}
-\sum_{k=1}^{m-2} \alpha_{k} \phi_{1}\left(\xi_{k}\right) & \sum_{k=1}^{m-2} \alpha_{k} \\
d-\sum_{k=1}^{m-2} \beta_{k} \phi_{1}\left(\xi_{k}\right) & \sum_{k=1}^{m-2} \beta_{k}
\end{array}\right| .
\end{aligned}
$$

\section{Main result}

In this section, we apply the following fixed point index theorem to prove the existence of at least one positive solution for the SSS (1).

Theorem 1. Let $E=(E,\|\|$.$) be a Banach space, \Omega$ be a bounded open subset of $E$ with $0 \in \Omega, P \subset E$ be a cone in $E$ and $F: P \cap \bar{\Omega} \rightarrow P$ be a completely continuous operator.

(i) Suppose that $F u \neq \lambda u, \quad \forall u \in \partial \Omega \cap P, \lambda \geq 1$. Then $i(F, \Omega \cap P, P)=1$.

(ii) Suppose that $F u \not \leq u, \forall u \in \partial \Omega \cap P$. Then $i(F, \Omega \cap P, P)=0$.

In the remaining part of the paper, we assume that the following conditions are satisfied:

$\left(H_{5}\right) f_{1}, f_{2}:(a, b) \times[0, \infty) \times[0, \infty) \rightarrow[0, \infty)$ are continuous,

$\left(H_{6}\right) h_{1}, h_{2}:(a, b) \rightarrow(-\infty, \infty)$ are Lebesgue integrable such that $0<\int_{a}^{b} G(s, s)\left[f_{i}(s, 1,1)+h_{i_{+}}(s)\right] \nabla s<\infty$ and $\int_{a}^{b} h_{i_{-}}(s) \nabla s>0 \quad i=1,2$,

where $h_{i_{+}}(t)=\max \left\{h_{i}(t), 0\right\}$ and $h_{i_{-}}(t)=\max \left\{-h_{i}(t), 0\right\}$.

Remark 1. By the assumption $\left(H_{6}\right)$, we have $\int_{a}^{b} G(t, s) h_{i_{-}}(s) \nabla s<\infty, \quad i=1,2$.

In fact, from the properties of $\phi_{1}, \phi_{2}$ and Green function, we get

$$
\int_{a}^{b} G(t, s) h_{i_{-}}(s) \nabla s \leq \int_{a}^{b} G(s, s) h_{i_{-}}(s) \nabla s \leq \phi_{1}(b) \phi_{2}(a) \int_{a}^{b} h_{i_{-}}(s) \nabla s<\infty, \quad i=1,2 .
$$

Let $w_{i}(t)=\int_{a}^{b} G(t, s) h_{i_{-}}(s) \nabla s+A\left(h_{i_{-}}\right) \phi_{1}(t)+B\left(h_{i_{-}}\right) \phi_{2}(t), t \in[a, b], i=1,2$. Using the expression for Green's function, the definition of the function $g$, the properties of $\phi_{1}$ and $\phi_{2}$, the assumption $\left(H_{6}\right)$ and Lemma 6 , we obtain

$$
\begin{aligned}
w_{i}(t)= & \int_{a}^{b} G(t, s) h_{i_{-}}(s) \nabla s+A\left(h_{i_{-}}\right) \phi_{1}(t)+B\left(h_{i_{-}}\right) \phi_{2}(t) \\
= & \frac{1}{d} \int_{a}^{t} \phi_{1}(s) \phi_{2}(t) h_{i_{-}}(s) \nabla s+\frac{1}{d} \int_{t}^{b} \phi_{1}(t) \phi_{2}(s) h_{i_{-}}(s) \nabla s+A\left(h_{i_{-}}\right) \phi_{1}(t)+B\left(h_{i_{-}}\right) \phi_{2}(t) \\
\leq & \frac{1}{d} \int_{a}^{t} \phi_{1}(t) \phi_{2}(t) h_{i_{-}}(s) \nabla s+\frac{1}{d} \int_{t}^{b} \phi_{1}(t) \phi_{2}(t) h_{i_{-}}(s) \nabla s+A \phi_{1}(t) \int_{a}^{b} G(s, s) h_{i_{-}}(s) \nabla s \\
& +B \phi_{2}(t) \int_{a}^{b} G(s, s) h_{i_{-}}(s) \nabla s \\
= & \frac{1}{d} \int_{a}^{b} \phi_{1}(t) \phi_{2}(t) h_{i_{-}}(s) \nabla s+A \phi_{1}(t) \int_{a}^{b} G(s, s) h_{i_{-}}(s) \nabla s+B \phi_{2}(t) \int_{a}^{b} G(s, s) h_{i_{-}}(s) \nabla s
\end{aligned}
$$




$$
\begin{aligned}
\leq & \frac{1}{d} \phi_{1}(b) \phi_{2}(a) g(t) \int_{a}^{b} h_{i_{-}}(s) \nabla s+\frac{1}{\phi_{2}(t)} A \phi_{1}(b) \phi_{2}(a) g(t) \int_{a}^{b} G(s, s) h_{i_{-}}(s) \nabla s \\
& +\frac{1}{\phi_{1}(t)} B \phi_{1}(b) \phi_{2}(a) g(t) \int_{a}^{b} G(s, s) h_{i_{-}}(s) \nabla s \\
\leq & \frac{1}{d} \phi_{1}(b) \phi_{2}(a) g(t) \int_{a}^{b} h_{i_{-}}(s) \nabla s+\frac{1}{\phi_{2}(b)} A \phi_{1}(b) \phi_{2}(a) g(t) \int_{a}^{b} G(s, s) h_{i_{-}}(s) \nabla s \\
& +\frac{1}{\phi_{1}(a)} B \phi_{1}(b) \phi_{2}(a) g(t) \int_{a}^{b} G(s, s) h_{i_{-}}(s) \nabla s \\
= & {\left[\frac{1}{d} \int_{a}^{b} h_{i_{-}}(s) \nabla s+\left(\frac{A}{\phi_{2}(b)}+\frac{B}{\phi_{1}(a)}\right) \int_{a}^{b} G(s, s) h_{i_{-}}(s) \nabla s\right] \phi_{1}(b) \phi_{2}(a) g(t)<+\infty, \quad i=1,2 . }
\end{aligned}
$$

Therefore, we can write

$$
w_{i}(t) \leq C_{i} g(t), \quad t \in[a, b], \quad i=1,2
$$

where

$$
C_{i}=\left[\frac{1}{d} \int_{a}^{b} h_{i_{-}}(s) \nabla s+\left(\frac{A}{\phi_{2}(b)}+\frac{B}{\phi_{1}(a)}\right) \int_{a}^{b} G(s, s) h_{i_{-}}(s) \nabla s\right] \phi_{1}(b) \phi_{2}(a)
$$

and $g$ is given in equation (2). Therefore, $w_{i}(t), i=1,2$ are well defined in $E$. By direct computation, we have

$$
\alpha w_{i}(a)-\beta w_{i}^{[\triangle]}(a)=\sum_{k=1}^{m-2} \alpha_{k} w_{i}\left(\xi_{k}\right), \quad \gamma w_{i}(b)+\delta w_{i}^{[\triangle]}(b)=\sum_{k=1}^{m-2} \beta_{k} w_{i}\left(\xi_{k}\right), \quad i=1,2,
$$

which implies that $w_{i}(t), i=1,2$ are positive solutions of the following boundary value problems:

$$
\begin{aligned}
& -\left[p(t) u_{1}^{\triangle}(t)\right]^{\nabla}+q(t) u_{1}(t)=h_{1_{-}}(t), \quad t \in(a, b), \\
& \alpha u_{1}(a)-\beta u_{1}^{[\triangle]}(a)=\sum_{k=1}^{m-2} \alpha_{k} u_{1}\left(\xi_{k}\right), \quad \gamma u_{1}(b)+\delta u_{1}^{[\triangle]}(b)=\sum_{k=1}^{m-2} \beta_{k} u_{1}\left(\xi_{k}\right),
\end{aligned}
$$

and

$$
\begin{aligned}
& -\left[p(t) u_{2}^{\triangle}(t)\right]^{\nabla}+q(t) u_{2}(t)=h_{2_{-}}(t), \quad t \in(a, b), \\
& \alpha u_{2}(a)-\beta u_{2}^{[\triangle]}(a)=\sum_{k=1}^{m-2} \alpha_{k} u_{2}\left(\xi_{k}\right), \quad \gamma u_{2}(b)+\delta u_{2}^{[\triangle]}(b)=\sum_{k=1}^{m-2} \beta_{k} u_{2}\left(\xi_{k}\right),
\end{aligned}
$$

respectively.

For any $u(t) \in C([a, b])$, let us define a function $[.]^{*}$ by

$$
[u(t)]^{*}= \begin{cases}u(t), & u(t) \geq 0 \\ 0 & u(t)<0\end{cases}
$$

Now, we consider the following dynamic system

$$
\left\{\begin{array}{l}
-\left[p(t) u_{i}^{\triangle}(t)\right]^{\nabla}+q(t) u_{i}(t)=f_{i}\left(t,\left[\left(u_{1}-w_{1}\right)(t)\right]^{*},\left[\left(u_{2}-w_{2}\right)(t)\right]^{*}\right)+h_{i_{+}}(t), \quad t \in(a, b), \quad i=1,2, \\
\alpha u_{i}(a)-\beta u_{i}^{[\Delta]}(a)=\sum_{k=1}^{m-2} \alpha_{k} u_{i}\left(\xi_{k}\right), \quad \gamma u_{i}(b)+\delta u_{i}^{[\triangle]}(b)=\sum_{k=1}^{m-2} \beta_{k} u_{i}\left(\xi_{k}\right), \quad i=1,2,
\end{array}\right.
$$

and we define the operator $F: E \rightarrow E$ by

$$
F\left(u_{1}, u_{2}\right)=\left(F_{1}\left(u_{1}, u_{2}\right), F_{2}\left(u_{1}, u_{2}\right)\right)
$$

where

$$
\begin{aligned}
F_{i}\left(u_{1}, u_{2}\right)(t)= & \int_{a}^{b} G(t, s)\left[f_{i}\left(s,\left[u_{1}(s)-w_{1}(s)\right]^{*},\left[u_{2}(s)-w_{2}(s)\right]^{*}\right)+h_{i_{+}}(s)\right] \nabla s+A\left(f_{i}+h_{i_{+}}\right) \phi_{1}(t) \\
& +B\left(f_{i}+h_{i_{+}}\right) \phi_{2}(t), \quad i=1,2 .
\end{aligned}
$$


It is well known that the existence of the solution to the system (7) is equivalent to the existence of fixed point of the operator $F$. Therefore, we shall seek a fixed point of $F$ in our cone $P$.

Lemma 7. If $\left(v_{1}, v_{2}\right)$ with $\left(w_{1}, w_{2}\right) \leq\left(v_{1}, v_{2}\right)$ is a positive solution of the system (7), then $\left(v_{1}-w_{1}, v_{2}-w_{2}\right)$ is a positive solution of the SSS (1).

Proof. Suppose that $\left(v_{1}, v_{2}\right)$ with $\left(w_{1}, w_{2}\right) \leq\left(v_{1}, v_{2}\right)$ is a positive solution of system (7), then from (7) and the definition of $[.]^{*}$, we have

$$
\begin{aligned}
& \left\{\begin{array}{l}
-\left[p(t) v_{i}^{\triangle}(t)\right]^{\nabla}+q(t) v_{i}(t)=f_{i}\left(t, v_{1}(t)-w_{1}(t), v_{2}(t)-w_{2}(t)\right)+h_{i_{+}}(t), \quad t \in(a, b), \quad i=1,2 \\
\alpha v_{i}(a)-\beta v_{i}^{[\triangle]}(a)=\sum_{k=1}^{m-2} \alpha_{k} v_{i}\left(\xi_{k}\right), \quad \gamma v_{i}(b)+\delta v_{i}^{[\triangle]}(b)=\sum_{k=1}^{m-2} \beta_{k} v_{i}\left(\xi_{k}\right), \quad i=1,2 .
\end{array}\right. \\
& \text { Let } u_{i}(t)=v_{i}(t)-w_{i}(t), i=1,2 \text {, then } v_{i}(t)=u_{i}(t)+w_{i}(t), v_{i}^{\triangle}(t)=\left(u_{i}+w_{i}\right) \triangle(t)=u_{i}^{\triangle}(t)+w_{i}^{\triangle}(t) \text { and } \\
& {\left[p(t) v_{i}^{\triangle}(t)\right]^{\nabla}=\left[p(t)\left(u_{i}^{\triangle}(t)+w_{i}^{\triangle}(t)\right)\right]^{\nabla}=\left[p(t) u_{i}^{\triangle}(t)\right]^{\nabla}+\left[p(t) w_{i}^{\triangle}(t)\right]^{\nabla},}
\end{aligned}
$$

thus (8) becomes

$$
\left\{\begin{array}{l}
-\left[p(t) u_{i}^{\triangle}(t)\right]^{\nabla}+q(t) u_{i}(t)=f_{i}\left(t, u_{1}(t), u_{2}(t)\right)+h_{i_{+}}(t)-h_{i_{-}}(t), \quad t \in(a, b), \quad i=1,2, \\
\alpha u_{i}(a)-\beta u_{i}^{[\triangle]}(a)=\sum_{k=1}^{m-2} \alpha_{k} u_{i}\left(\xi_{k}\right), \quad \gamma u_{i}(b)+\delta u_{i}^{[\triangle]}(b)=\sum_{k=1}^{m-2} \beta_{k} u_{i}\left(\xi_{k}\right), \quad i=1,2,
\end{array}\right.
$$

Notice that $h_{i}(t)=h_{i_{+}}(t)-h_{i_{-}}(t), i=1,2$ and (9). We know that $\left(u_{1}, u_{2}\right)=\left(v_{1}-w_{1}, v_{2}-w_{2}\right)$ is a positive solution of the SSS (1). This completes the proof.

Now, we want to give the main result of this paper. To prove the main theorem, we need the following assumptions for the functions $f_{i}, \mathrm{i}=1,2$.

$\left(H_{7}\right)$ For $t \in(a, b), f_{i}(t, 1,1) \neq 0(i=1,2)$, there exists constants $\lambda_{1} \geq \lambda_{2}>1$ such that, for $t \in(a, b)$, $u_{1}, u_{2} \in[0, \infty)$,

$$
\left(c_{1} c_{2}\right)^{\lambda_{1}} f_{i}\left(t, u_{1}, u_{2}\right) \leq f_{i}\left(t, c_{1} u_{1}, c_{2} u_{2}\right) \leq\left(c_{1} c_{2}\right)^{\lambda_{2}} f_{i}\left(t, u_{1}, u_{2}\right), \quad \forall c_{1}, c_{2} \in[0,1], \quad i=1,2 .
$$

Remark 2. For $c_{1}, c_{2} \geq 1,\left(t, u_{1}, u_{2}\right) \in(a, b) \times[0, \infty) \times[0, \infty)$, we have

$$
\left(c_{1} c_{2}\right)^{\lambda_{2}} f_{i}\left(t, u_{1}, u_{2}\right) \leq f_{i}\left(t, c_{1} u_{1}, c_{2} u_{2}\right) \leq\left(c_{1} c_{2}\right)^{\lambda_{1}} f_{i}\left(t, u_{1}, u_{2}\right), \quad i=1,2 .
$$

In fact, from the assumption $\left(H_{7}\right)$, for $c_{1}, c_{2} \geq 1,\left(t, u_{1}, u_{2}\right) \in(a, b) \times[0, \infty) \times[0, \infty)$, we get

$$
f_{i}\left(t, u_{1}, u_{2}\right)=f_{i}\left(t, \frac{1}{c_{1}} c_{1} u_{1}, \frac{1}{c_{2}} c_{2} u_{2}\right) \leq\left(\frac{1}{c_{1} c_{2}}\right)^{\lambda_{2}} f_{i}\left(t, c_{1} u_{1}, c_{2} u_{2}\right)
$$

This implies

$$
\left(c_{1} c_{2}\right)^{\lambda_{2}} f_{i}\left(t, u_{1}, u_{2}\right) \leq f_{i}\left(t, c_{1} u_{1}, c_{2} u_{2}\right), \quad i=1,2 .
$$

At the same time, we have

$$
f_{i}\left(t, c_{1} u_{1}, c_{2} u_{2}\right) \leq\left(c_{1} c_{2}\right)^{\lambda_{1}} f_{i}\left(t, u_{1}, u_{2}\right), \quad i=1,2 .
$$

Therefore, when $c_{1}, c_{2} \geq 1$, we have

$$
\left(c_{1} c_{2}\right)^{\lambda_{2}} f_{i}\left(t, u_{1}, u_{2}\right) \leq f_{i}\left(t, c_{1} u_{1}, c_{2} u_{2}\right) \leq\left(c_{1} c_{2}\right)^{\lambda_{1}} f_{i}\left(t, u_{1}, u_{2}\right), \quad i=1,2 .
$$

Lemma 8. If $f_{i}\left(t, u_{1}, u_{2}\right)(i=1,2)$ satisfies $\left(H_{7}\right)$, then for $\left(t, u_{1}, u_{2}\right) \in(a, b) \times[0, \infty) \times[0, \infty), f_{i}\left(t, u_{1}, u_{2}\right)$ is increasing on $u_{1}, u_{2}$ and for $\left[t_{1}, t_{2}\right] \subset(a, b)$,

$$
\lim _{u_{1}, u_{2} \rightarrow+\infty} \min _{t \in\left[t_{1}, t_{2}\right]} \frac{f_{i}\left(t, u_{1}, u_{2}\right)}{\left|u_{1}\right|+\left|u_{2}\right|}=+\infty, \quad i=1,2 .
$$


Proof. Let $t \in(a, b), u_{1}, v_{1}, u_{2} \in[0, \infty)$ such that $u_{1} \leq v_{1}$. We will show that $f_{i}\left(t, u_{1}, u_{2}\right) \leq f_{i}\left(t, v_{1}, u_{2}\right)(i=1,2)$. Clearly, if $v_{1}=0$, then $f_{i}\left(t, u_{1}, u_{2}\right) \leq f_{i}\left(t, v_{1}, u_{2}\right)$. If $v_{1} \neq 0$, let $a_{1}=u_{1} / v_{1}$, then $0 \leq a_{1} \leq 1$. Now, using the assumption $\left(H_{7}\right)$, we obtain

$$
f_{i}\left(t, u_{1}, u_{2}\right)=f_{i}\left(t, a_{1} v_{1}, u_{2}\right) \leq a_{1}^{\lambda_{2}} f_{i}\left(t, v_{1}, u_{2}\right) \leq f_{i}\left(t, v_{1}, u_{2}\right), \quad i=1,2 .
$$

Thus, we get that $f_{i}\left(t, u_{1}, u_{2}\right)$ is increasing on $u_{1}$. Similarly, we can prove that $f_{i}\left(t, u_{1}, u_{2}\right)$ is increasing on $u_{2}$. On the other hand, choose $u_{1}, u_{2}>1$. Considering the Remark 2, we get

$$
f_{i}\left(t, u_{1}, u_{2}\right) \geq\left(u_{1} u_{2}\right)^{\lambda_{2}} f_{i}(t, 1,1), \quad i=1,2,
$$

and thus, for $\left[t_{1}, t_{2}\right] \subset(a, b), \forall t \in\left[t_{1}, t_{2}\right]$, we have

$$
\min _{t \in\left[t_{1}, t_{2}\right]} \frac{f_{i}\left(t, u_{1}, u_{2}\right)}{\left|u_{1}\right|+\left|u_{2}\right|} \geq \min _{t \in\left[t_{1}, t_{2}\right]} \frac{\left(u_{1} u_{2}\right)^{\lambda_{2}}}{\left|u_{1}\right|+\left|u_{2}\right|} f_{i}(t, 1,1)>0,
$$

Therefore, we obtain

$$
\lim _{u_{1}, u_{2} \rightarrow+\infty} \min _{t \in\left[t_{1}, t_{2}\right]} \frac{f_{i}\left(t, u_{1}, u_{2}\right)}{\left|u_{1}\right|+\left|u_{2}\right|}=+\infty, \quad i=1,2
$$

Lemma 9. Assume that $\left(H_{1}\right)-\left(H_{7}\right)$ hold. Then $F: P \rightarrow P$ is a completely continuous operator.

Proof. First, we shall show that the operator $F: P \rightarrow P$ is well defined. Therefore, for any fixed $\left(u_{1}, u_{2}\right) \in P$, choose $0<d_{1}, d_{2}<1$ such that $d_{1}\left\|u_{1}\right\|<1$ and $d_{2}\left\|u_{2}\right\|<1$. Then for $t \in[a, b]$, we get

$$
d_{i}\left[u_{i}(t)-w_{i}(t)\right]^{*} \leq d_{i} u_{i}(t) \leq d_{i}\left\|u_{i}\right\|<1, \quad i=1,2 .
$$

Thus, using Remark 2 and Lemma 8, we get

$$
\begin{aligned}
f_{i}\left(t,\left[u_{1}(t)-w_{1}(t)\right]^{*},\left[u_{2}(t)-w_{2}(t)\right]^{*}\right) & \leq\left(\frac{1}{d_{1} d_{2}}\right)^{\lambda_{1}} f_{i}\left(t, d_{1}\left\|u_{1}\right\|, d_{2}\left\|u_{2}\right\|\right) \\
& \leq\left(d_{1} d_{2}\right)^{\lambda_{2}-\lambda_{1}}\left\|u_{1}\right\|^{\lambda_{2}}\left\|u_{2}\right\|^{\lambda_{2}} f_{i}(t, 1,1), \quad i=1,2
\end{aligned}
$$

from which the assumption $\left(H_{6}\right)$, the properties of $\phi_{1}, \phi_{2}$ and Lemma 6 , for any $t \in[a, b]$ gives us:

$$
\begin{aligned}
F_{i}\left(u_{1}, u_{2}\right)(t)= & \int_{a}^{b} G(t, s)\left[f_{i}\left(s,\left[u_{1}(s)-w_{1}(s)\right]^{*},\left[u_{2}(s)-w_{2}(s)\right]^{*}\right)+h_{i_{+}}(s)\right] \nabla s \\
& +A\left(f_{i}+h_{i_{+}}\right) \phi_{1}(t)+B\left(f_{i}+h_{i_{+}}\right) \phi_{2}(t) \\
\leq & \int_{a}^{b} G(s, s)\left[f_{i}\left(s,\left[u_{1}(s)-w_{1}(s)\right]^{*},\left[u_{2}(s)-w_{2}(s)\right]^{*}\right)+h_{i_{+}}(s)\right] \nabla s \\
& +A\left(f_{i}+h_{i_{+}}\right) \phi_{1}(t)+B\left(f_{i}+h_{i_{+}}\right) \phi_{2}(t) \\
\leq & \int_{a}^{b} G(s, s)\left[\left(d_{1} d_{2}\right)^{\lambda_{2}-\lambda_{1}}\left\|u_{1}\right\|^{\lambda_{2}}\left\|u_{2}\right\|^{\lambda_{2}} f_{i}(s, 1,1)+h_{i_{+}}(s)\right] \nabla s \\
& +A\left(f_{i}+h_{i_{+}}\right) \phi_{1}(b)+B\left(f_{i}+h_{i_{+}}\right) \phi_{2}(a) \\
\leq & \left(\left(d_{1} d_{2}\right)^{\lambda_{2}-\lambda_{1}}\left\|u_{1}\right\|^{\lambda_{2}}\left\|u_{2}\right\|^{\lambda_{2}}+1\right) \int_{a}^{b} G(s, s)\left[f_{i}(s, 1,1)+h_{i_{+}}(s)\right] \nabla s \\
& +A\left(\left(d_{1} d_{2}\right)^{\lambda_{2}-\lambda_{1}}\left\|u_{1}\right\|^{\lambda_{2}}\left\|u_{2}\right\|^{\lambda_{2}}+1\right) \phi_{1}(b) \int_{a}^{b} G(s, s)\left[f_{i}(s, 1,1)+h_{i_{+}}(s)\right] \nabla s \\
& +B\left(\left(d_{1} d_{2}\right)^{\lambda_{2}-\lambda_{1}}\left\|u_{1}\right\|^{\lambda_{2}}\left\|u_{2}\right\|^{\lambda_{2}}+1\right) \phi_{2}(a) \int_{a}^{b} G(s, s)\left[f_{i}(s, 1,1)+h_{i_{+}}(s)\right] \nabla s \\
= & \left(\left(d_{1} d_{2}\right)^{\lambda_{2}-\lambda_{1}}\left\|u_{1}\right\|^{\lambda_{2}}\left\|u_{2}\right\|^{\lambda_{2}}+1\right)\left(1+A \phi_{1}(b)+B \phi_{2}(a)\right) \int_{a}^{b} G(s, s)\left[f_{i}(s, 1,1)+h_{i_{+}}(s)\right] \nabla s \\
< & \infty, \quad i=1,2 .
\end{aligned}
$$


Thus $F: P \rightarrow E$ is well defined. Now we shall prove that $F(P) \subseteq P$. For any $\left(u_{1}, u_{2}\right) \in P$, let $\left(v_{1}(t), v_{2}(t)\right)=F\left(u_{1}, u_{2}\right)(t)$. Then for $t \in[a, b]$, we get

$$
\begin{aligned}
v_{i}(t) & =\int_{a}^{b} G(t, s)\left[f_{i}\left(s,\left[u_{1}(s)-w_{1}(s)\right]^{*},\left[u_{2}(s)-w_{2}(s)\right]^{*}\right)+h_{i_{+}}(s)\right] \nabla s+A\left(f_{i}+h_{i_{+}}\right) \phi_{1}(t)+B\left(f_{i}+h_{i_{+}}\right) \phi_{2}(t) \\
& \leq \int_{a}^{b} G(s, s)\left[f_{i}\left(s,\left[u_{1}(s)-w_{1}(s)\right]^{*},\left[u_{2}(s)-w_{2}(s)\right]^{*}\right)+h_{i_{+}}(s)\right] \nabla s+A\left(f_{i}+h_{i_{+}}\right) \phi_{1}(b)+B\left(f_{i}+h_{i_{+}}\right) \phi_{2}(a)
\end{aligned}
$$

and so

$$
\left\|v_{i}\right\| \leq \int_{a}^{b} G(s, s)\left[f_{i}\left(s,\left[u_{1}(s)-w_{1}(s)\right]^{*},\left[u_{2}(s)-w_{2}(s)\right]^{*}\right)+h_{i_{+}}(s)\right] \nabla s+A\left(f_{i}+h_{i_{+}}\right) \phi_{1}(b)+B\left(f_{i}+h_{i_{+}}\right) \phi_{2}(a),
$$

For $t \in[a, b]$, the above relation and Lemma 3 gives:

$$
\begin{aligned}
v_{i}(t)= & \int_{a}^{b} G(t, s)\left[f_{i}\left(s,\left[u_{1}(s)-w_{1}(s)\right]^{*},\left[u_{2}(s)-w_{2}(s)\right]^{*}\right)+h_{i_{+}}(s)\right] \nabla s+A\left(f_{i}+h_{i_{+}}\right) \phi_{1}(t)+B\left(f_{i}+h_{i_{+}}\right) \phi_{2}(t) \\
\geq & g(t) \int_{a}^{b} G(s, s)\left[f_{i}\left(s,\left[u_{1}(s)-w_{1}(s)\right]^{*},\left[u_{2}(s)-w_{2}(s)\right]^{*}\right)+h_{i_{+}}(s)\right] \nabla s \\
& +A\left(f_{i}+h_{i_{+}}\right) \frac{\phi_{1}(t)}{\phi_{1}(b)} \phi_{1}(b)+B\left(f_{i}+h_{i_{+}}\right) \frac{\phi_{2}(t)}{\phi_{2}(a)} \phi_{2}(a) \\
\geq & g(t) \int_{a}^{b} G(s, s)\left[f_{i}\left(s,\left[u_{1}(s)-w_{1}(s)\right]^{*},\left[u_{2}(s)-w_{2}(s)\right]^{*}\right)+h_{i_{+}}(s)\right] \nabla s \\
& +A\left(f_{i}+h_{i_{+}}\right) g(t) \phi_{1}(b)+B\left(f_{i}+h_{i_{+}}\right) g(t) \phi_{2}(a) \\
= & g(t)\left[\int_{a}^{b} G(s, s)\left[f_{i}\left(s,\left[u_{1}(s)-w_{1}(s)\right]^{*},\left[u_{2}(s)-w_{2}(s)\right]^{*}\right)+h_{i_{+}}(s)\right] \nabla s\right. \\
& \left.+A\left(f_{i}+h_{i_{+}}\right) \phi_{1}(b)+B\left(f_{i}+h_{i_{+}}\right) \phi_{2}(a)\right] \\
\geq & g(t)\left\|v_{i}\right\|, \quad i=1,2 .
\end{aligned}
$$

This yields that $F(P) \subseteq P$.

Let $D \subset P$ be any bounded set. Then there exists a constant $M>0$ such that $\left\|u_{i}\right\| \leq M, \quad i=1,2$ for any $\left(u_{1}, u_{2}\right) \in D$. Furthermore for any $\left(u_{1}, u_{2}\right) \in D$ and $t \in[a, b]$, we find

$$
0 \leq\left[u_{i}(t)-w_{i}(t)\right]^{*} \leq u_{i}(t) \leq\left\|u_{i}\right\| \leq M<M+1, \quad i=1,2 .
$$

Thus, by Remark 2 and Lemma 8, for any $s \in[a, b]$, we have

$$
\begin{aligned}
& f_{i}\left(s,\left[u_{1}(s)-w_{1}(s)\right]^{*},\left[u_{2}(s)-w_{2}(s)\right]^{*}\right)+h_{i_{+}}(s) \leq f_{i}(s, M+1, M+1)+h_{i_{+}}(s) \\
& \leq(M+1)^{2 \lambda_{1}} f_{i}(s, 1,1)+h_{i_{+}}(s) \leq\left((M+1)^{2 \lambda_{1}}+1\right)\left[f_{i}(s, 1,1)+h_{i_{+}}(s)\right], \quad i=1,2 .
\end{aligned}
$$

Consequently,

$$
\begin{aligned}
F_{i}\left(u_{1}, u_{2}\right)(t)= & \int_{a}^{b} G(t, s)\left[f_{i}\left(s,\left[u_{1}(s)-w_{1}(s)\right]^{*},\left[u_{2}(s)-w_{2}(s)\right]^{*}\right)+h_{i_{+}}(s)\right] \nabla s+A\left(f_{i}+h_{i_{+}}\right) \phi_{1}(t)+B\left(f_{i}+h_{i_{+}}\right) \phi_{2}(t) \\
\leq & \int_{a}^{b} G(s, s)\left((M+1)^{2 \lambda_{1}}+1\right)\left[f_{i}(s, 1,1)+h_{i_{+}}(s)\right] \nabla s \\
& +A \phi_{1}(b) \int_{a}^{b} G(s, s)\left((M+1)^{2 \lambda_{1}}+1\right)\left[f_{i}(s, 1,1)+h_{i_{+}}(s)\right] \nabla s \\
& +B \phi_{2}(a) \int_{a}^{b} G(s, s)\left((M+1)^{2 \lambda_{1}}+1\right)\left[f_{i}(s, 1,1)+h_{i_{+}}(s)\right] \nabla s \\
= & \left((M+1)^{2 \lambda_{1}}+1\right)\left(1+A \phi_{1}(b)+B \phi_{2}(a)\right) \int_{a}^{b} G(s, s)\left[f_{i}(s, 1,1)+h_{i_{+}}(s)\right] \nabla s \\
< & \infty, \quad i=1,2 .
\end{aligned}
$$

Therefore $F(D)$ is uniformly bounded. 
Similarly, we can easily find $F(D)$ is equicontinuous on $[a, b]$. Thus from the Ascoli-Arzela Theorem, we know that $F(D)$ is a relatively compact set.

Finally, from the continuity of $f_{i}, i=1,2$, it is not difficult to check that $F: P \rightarrow P$ is continuous. Hence $F: P \rightarrow P$ is a completely continuous operator.

Theorem 2. Let $\left(H_{1}\right)-\left(H_{7}\right)$ hold. For each $r$ satisfying

$$
r>\max \left\{2 C_{1}, 2 C_{2},\left((r+1)^{2 \lambda_{1}}+1\right)\left(1+A \phi_{1}(b)+B \phi_{2}(a)\right) \int_{a}^{b} G(s, s)\left[f_{i}(s, 1,1)+h_{i_{+}}(s)\right] \nabla s\right\},
$$

where $C_{i}(i=1,2)$ are given in (6). The SSS (1) has at least one positive solution $\left(\tilde{u_{1}}, \tilde{u_{2}}\right)$ such that $\left\|\tilde{u}_{i}\right\|>r, i=1,2$.

Proof. Assume that there exist $\lambda_{0} \geq 1$ and $\left(\tilde{u_{1}}, \tilde{u_{2}}\right) \in \partial P_{r}$ such that $F\left(\tilde{u_{1}}, \tilde{u_{2}}\right)=\lambda_{0}\left(\tilde{u}_{1}, \tilde{u}_{2}\right)$ where

$P_{r}=\left\{\left(u_{1}, u_{2}\right) \in P:\left\|u_{1}\right\|<r,\left\|u_{2}\right\|<r\right\}$. Then $\frac{1}{\lambda_{0}}\left(F_{1}\left(\tilde{u_{1}}, \tilde{u_{2}}\right), F_{2}\left(\tilde{u_{1}}, \tilde{u_{2}}\right)\right)=\left(\tilde{u_{1}}, \tilde{u_{2}}\right)$ and $0<\frac{1}{\lambda_{0}} \leq 1$. Moreover for $t \in[a, b]$, we obtain

$$
0 \leq\left[\tilde{u}_{i}(t)-w_{i}(t)\right]^{*} \leq \tilde{u}_{i}(t) \leq\left\|\tilde{u}_{i}\right\|=r<r+1, \quad i=1,2,
$$

from which, using Remark 2 and Lemma 8 , for $t \in[a, b]$, we get

$$
f_{i}\left(s,\left[u_{1}(s)-w_{1}(s)\right]^{*},\left[u_{2}(s)-w_{2}(s)\right]^{*}\right) \leq f_{i}(s, r+1, r+1) \leq(r+1)^{2 \lambda_{1}} f_{i}(s, 1,1), \quad i=1,2 .
$$

Now, using Lemma 1 and Lemma 6 and the properties of the operators $A, B$, for $t \in[a, b]$, we get

$$
\begin{aligned}
\tilde{u_{i}}(t)= & \frac{1}{\lambda_{0}}\left\{\int_{a}^{b} G(t, s)\left[f_{i}\left(s,\left[\tilde{u_{1}}(s)-w_{1}(s)\right]^{*},\left[\tilde{u}_{2}(s)-w_{2}(s)\right]^{*}\right)+h_{i_{+}}(s)\right] \nabla s\right. \\
& \left.+A\left(f_{i}+h_{i_{+}}\right) \phi_{1}(t)+B\left(f_{i}+h_{i_{+}}\right) \phi_{2}(t)\right\} \\
\leq & \int_{a}^{b} G(s, s)\left[f_{i}\left(s,\left[\tilde{u}_{1}(s)-w_{1}(s)\right]^{*},\left[\tilde{u}_{2}(s)-w_{2}(s)\right]^{*}\right)+h_{i_{+}}(s)\right] \nabla s+A\left(f_{i}+h_{i_{+}}\right) \phi_{1}(t)+B\left(f_{i}+h_{i_{+}}\right) \phi_{2}(t) \\
\leq & \int_{a}^{b} G(s, s)\left((r+1)^{2 \lambda_{1}}+1\right)\left[f_{i}(s, 1,1)+h_{i_{+}}(s)\right] \nabla s \\
& +A \phi_{1}(b) \int_{a}^{b} G(s, s)\left((r+1)^{2 \lambda_{1}}+1\right)\left[f_{i}(s, 1,1)+h_{i_{+}}(s)\right] \nabla s \\
& +B \phi_{2}(a) \int_{a}^{b} G(s, s)\left((r+1)^{2 \lambda_{1}}+1\right)\left[f_{i}(s, 1,1)+h_{i_{+}}(s)\right] \nabla s \\
= & \left((r+1)^{2 \lambda_{1}}+1\right)\left(1+A \phi_{1}(b)+B \phi_{2}(a)\right) \int_{a}^{b} G(s, s)\left[f_{i}(s, 1,1)+h_{i_{+}}(s)\right] \nabla s .
\end{aligned}
$$

Thus, we get

$$
r \leq\left((r+1)^{2 \lambda_{1}}+1\right)\left(1+A \phi_{1}(b)+B \phi_{2}(a)\right) \int_{a}^{b} G(s, s)\left[f_{i}(s, 1,1)+h_{i_{+}}(s)\right] \nabla s, \quad i=1,2 .
$$

This is a contradiction. Then by Theorem 1, we have

$$
i\left(F, P_{r}, P\right)=1 .
$$

On the other hand, let us choose the constant $K$ such that

$$
K>\left(\inf _{t \in\left[t_{1}, t_{2}\right]} g(t) \max _{t \in[a, b]} \int_{t_{1}}^{t_{2}} G(t, s) \nabla s\right)^{-1} .
$$

In view of Lemma 8 , there exists $N>0$ such that

$$
f_{i}\left(t, u_{1}, u_{2}\right) \geq K\left(u_{1}+u_{2}\right), \quad u_{1} \geq N, u_{2} \geq N \quad \text { and } \quad t \in\left[t_{1}, t_{2}\right], \quad i=1,2 .
$$

Now, set

$$
R=r+2 N\left(\inf _{t \in\left[t_{1}, t_{2}\right]} g(t)\right)^{-1}
$$


Next, we show that $F\left(u_{1}, u_{2}\right) \not \leq\left(u_{1}, u_{2}\right)$ for any $\left(u_{1}, u_{2}\right) \in \partial P_{R}$. In fact, otherwise, there exists $\left(\hat{u}_{1}, \hat{u}_{2}\right) \in$ $\partial P_{R}$ such that $\left(\hat{u_{1}}, \hat{u_{2}}\right) \geq F\left(\hat{u}_{1}, \hat{u_{2}}\right)=\left(F_{1}\left(\hat{u_{1}}, \hat{u_{2}}\right), F_{2}\left(\hat{u_{1}}, \hat{u_{2}}\right)\right)$. From $(6)$ and the fact that $\left(\hat{u_{1}}, \hat{u_{2}}\right) \in \partial P_{R}$, for $t \in[a, b]$, we get

$$
w_{i}(t) \leq C_{i} g(t) \leq C_{i} \frac{\hat{u}_{i}(t)}{R} \quad i=1,2,
$$

and noting that $R>r>\max \left\{2 C_{1}, 2 C_{2}\right\}$, from (11) and (12), for all $t \in\left[t_{1}, t_{2}\right]$, we obtain

$$
\hat{u}_{i}(t)-w_{i}(t) \geq\left(1-\frac{C_{i}}{R}\right) \hat{u}_{i}(t) \geq \frac{1}{2} \hat{u}_{i}(t) \geq \frac{1}{2} g(t) R \geq \frac{R}{2} \inf _{t \in\left[t_{1}, t_{2}\right]} g(t)>N>0, \quad i=1,2 .
$$

Considering this, for $t \in[a, b]$, we get

$$
\begin{aligned}
\hat{u}_{i}(t) \geq & \int_{a}^{b} G(t, s)\left[f_{i}\left(s,\left[\hat{u}_{1}(s)-w_{1}(s)\right]^{*},\left[\hat{u}_{2}(s)-w_{2}(s)\right]^{*}\right)+h_{i_{+}}(s)\right] \nabla s \\
& +A\left(f_{i}+h_{i_{+}}\right) \phi_{1}(t)+B\left(f_{i}+h_{i_{+}}\right) \phi_{2}(t) \\
\geq & \int_{t_{1}}^{t_{2}} G(t, s) f_{i}\left(s,\left[\hat{u}_{1}(s)-w_{1}(s)\right]^{*},\left[\hat{u}_{2}(s)-w_{2}(s)\right]^{*}\right) \\
= & \int_{t_{1}}^{t_{2}} G(t, s) f_{i}\left(s, \hat{u}_{1}(s)-w_{1}(s), \hat{u}_{2}(s)-w_{2}(s)\right) \nabla s \\
\geq & \int_{t_{1}}^{t_{2}} G(t, s) K\left(\hat{u}_{1}(s)-w_{1}(s)+\hat{u}_{2}(s)-w_{2}(s)\right) \nabla s \\
\geq & \int_{t_{1}}^{t_{2}} G(t, s) K R \inf _{t \in\left[t_{1}, t_{2}\right]} g(t) \nabla s
\end{aligned}
$$

and so

$$
R \geq K R \inf _{t \in\left[t_{1}, t_{2}\right]} g(t) \max _{t \in[a, b]} \int_{t_{1}}^{t_{2}} G(t, s) \nabla s
$$

That is

$$
K \leq\left(\inf _{t \in\left[t_{1}, t_{2}\right]} g(t) \max _{t \in[a, b]} \int_{t_{1}}^{t_{2}} G(t, s) \nabla s\right)^{-1} .
$$

This contradicts the $K$ that we choose. So from Theorem 1 , we get

$$
i\left(F, P_{R}, P\right)=0 .
$$

Therefore, by equations (10) and (13), we have $i\left(F, P_{R} \backslash \overline{P_{r}}, P\right)=-1$.

Then we see that the operator $F$ has a fixed point $\left(\tilde{u_{1}}, \tilde{u_{2}}\right)$ in $P$ such that

$$
r<\left\|\tilde{u}_{i}\right\|<R, \quad i=1,2 .
$$

Moreover, using this and inequality (6) for $t \in[a, b]$, we get

$$
\tilde{u}_{i}(t) \geq g(t)\left\|\tilde{u}_{i}\right\|>\operatorname{rg}(t)>2 C_{i} g(t) \geq 2 w_{i}(t), \quad i=1,2 .
$$

Hence, $\left(\tilde{u_{1}}, \tilde{u_{2}}\right)$ with $\left(w_{1}, w_{2}\right) \leq\left(\tilde{u_{1}}, \tilde{u_{2}}\right)$ is a positive solution of the system (7). Therefore, by Lemma 7 , $\left(\tilde{u_{1}}-w_{1}, \tilde{u_{2}}-w_{2}\right)$ is the positive solution of the SSS (1).

Example 1. Let $\mathrm{T}=\left\{2^{k}: k \in Z\right\} \cup\{0\}$. Consider the following SSS,

$$
\left\{\begin{array}{l}
-u_{i}^{\triangle \nabla}(t)=f_{i}\left(t, u_{1}(t), u_{2}(t)\right)+h_{i}(t), \quad t \in(0,1), \quad i=1,2, \\
u_{i}(0)-u_{i}^{\triangle}(0)=u_{i}(1)+u_{i}^{\triangle}(1)=0, \quad i=1,2
\end{array}\right.
$$

where

$$
\begin{aligned}
& f_{1}\left(t, u_{1}, u_{2}\right)=t^{2}(1-t) u_{1}^{3 / 2} u_{2}^{2}+\sqrt{u_{1}}, \quad h_{1}(t)=-t \\
& f_{2}\left(t, u_{1}, u_{2}\right)=\frac{1}{10^{3} t(1-t)} u_{1}^{3 / 2}+\frac{1}{10^{2}} \sqrt{u_{1}+u_{2}}, \quad h_{2}(t)=-t^{2}
\end{aligned}
$$


Clearly $f_{1}$ and $f_{2}$ satisfy the condition $\left(H_{7}\right)$. We can easily calculate the followings;

$$
\begin{aligned}
& \int_{0}^{1} G(s, s) h_{1_{-}}(s) \nabla s=\int_{0}^{1} G(s, s) s \nabla s=\frac{1}{3} \int_{0}^{1}(1+s)(2-s) s \nabla s=\frac{16}{35}, \\
& \int_{0}^{1} G(s, s) h_{2_{-}}(s) \nabla s=\int_{0}^{1} G(s, s) s^{2} \nabla s=\frac{1}{3} \int_{0}^{1}(1+s)(2-s) s^{2} \nabla s=\frac{3776}{9765}, \\
& \int_{0}^{1} h_{1_{-}}(s) \nabla s=\int_{0}^{1} s \nabla s=\frac{2}{3}, \quad \int_{0}^{1} h_{2_{-}}(s) \nabla s=\int_{0}^{1} s^{2} \nabla s=\frac{4}{7}, \\
& \int_{0}^{1} G(s, s)\left(1+h_{i_{+}}(s)\right) \nabla s=\frac{1}{3} \int_{0}^{1}(1+s)(2-s) \nabla s=\frac{44}{63} \text { for } i=1,2, \\
& C_{1}=\frac{1}{3} \int_{0}^{1} h_{1_{-}}(s) \nabla s \phi_{1}(1) \phi_{2}(0)=\frac{8}{9}, \quad C_{2}=\frac{1}{3} \int_{0}^{1} h_{2_{-}}(s) \nabla s \phi_{1}(1) \phi_{2}(0)=\frac{16}{21},
\end{aligned}
$$

And for $\left(t, u_{1}, u_{2}\right) \in[0,1] \times[0, r] \times[0, r]$

$$
\begin{aligned}
& \left.K_{1}=\max \left\{t^{2}(1-t) u_{1}^{3 / 2} u_{2}^{2}+\sqrt{u_{1}}+1\right)\right\}=\frac{1}{12} r^{7 / 2}+\sqrt{r}+1 \\
& K_{2}=\max \left\{\frac{1}{10^{3} t(1-t)} u_{1}^{3 / 2}+\frac{1}{10^{2}} \sqrt{u_{1}+u_{2}}+1\right\}=\frac{1}{250} r^{3 / 2}+\frac{1}{10^{2}} \sqrt{2 r}+1
\end{aligned}
$$

If we choose $r=\frac{17}{9}$, we have

$$
r>\max \left\{\frac{16}{9}, \frac{32}{21}, K_{1} \frac{44}{63}, K_{2} \frac{44}{63}\right\} .
$$

Then, by Theorem 2 , the dynamic system has two positive solutions $\left(\tilde{u_{1}}, \tilde{u}_{2}\right)$ and $\left(\hat{u}_{1}, \hat{u_{2}}\right)$ such that

$$
0<\left\|\hat{u}_{i}\right\|<\frac{17}{9}<\left\|\tilde{u}_{i}\right\|, \quad i=1,2 .
$$

Author Contributions: Both authors contributed equally to the writing of this paper. All authors read and approved the final manuscript.

Conflicts of Interest: "The authors declare no conflict of interest."

\section{References}

[1] Joao, M., Sebastian, L., \& Pedro, U. (2005). Local superlinearity for elliptic system involving parameters. Journal of Differential Equations, 211, 1-19.

[2] Ma, R. (2000). Multiple nonnegative solutions of second-order systems of boundary value problems. Nonlinear Analysis, 42, 1003-1010.

[3] Wang, H. (2002). Multiplicity of positive radical solutions for an elliptic systems on an annulus. Nonlinear Analysis, $42,803-811$.

[4] Wang, H. (2003). On the number of positive solutions of nonlinear systems. Journal of Mathematical Analysis and Applications, 281, 287-306.

[5] Zhou, Y., \& Xu, Y. (2006). Positive solutions of three-point boundary value problems for systems of nonlinear second order ordinary differential equations. Journal of Mathematical Analysis and Applications, 320, 78-590.

[6] Asif, N.A., \& Khan, R.A. (2012). Positive solutions to singular system with four-point coupled boundary conditions. Journal of Mathematical Analysis and Applications, 386, 848-861.

[7] Liu, Y., \& Yan, B. (2003). Multiple solutions of singular boundary value problems for differential systems. Journal of Mathematical Analysis and Applications, 287, 540-556.

[8] Liu, L., Zhang, X., \& Wu, Y. (2007). On existence of positive solutions of a two-point boundary value problem for a nonlinear singular semipositone system. Applied Mathematics and Computation, 192, 223-232.

[9] Yang, Y., \& Meng, F. (2010). Positive solutions for the singular semipositone boundary value problem on time scales. Mathematical and Computer Modelling, 52, 481-489.

[10] Anderson, R., \& Wong, P. J. Y. (2009). Positive solutions for second-order semipositone problems on time scales. Computers and Mathematics with Applications, 58, 281-291.

[11] Anderson, D. R., \& Zhai, C. (2010). Positive solutions to semi-positone second-order three-point problems on time scales. Appl. Mathematics of Computation, 215, 3713-3720.

[12] Karaca, I. Y. (2008). Multiple positive solutions for dynamic m-point boundary value problems. Dynamic Systems and Applications, 17, 25-42. 
[13] Zhao, J., Lian, H., \& Ge, W. (2012). Existence of positive solutions for nonlinear m -point boundary value problems on time scales. Boundary Value Problems, 4, 15 pp.

[14] Cetin, E., \& Topal, S. G. (2010). Existence of multiple positive solutions for the system of higher order boundary value problems on time scales. Mathematical and Computer Modelling, 52, 1-11.

[15] Li, W.T., \& Sun, H.R. (2004). Multiple positive solutions for nonlinear dynamical systems on a measure chain. Journal of Computational and Applied Mathematics, 162, 421-430.

[16] Prasad, K.R., Rao, A.K., \& Bharathi, B. (2014). Positive solutions for system of 2n-th order Sturm Liouville boundary value problems on time scales. Proceedings of Indian Acadamy of Sciences (Mathematical Sciences), 124, 67-79.

[17] Aris, R. (1965). Introduction to the Analysis of Chemical Reactors. Prentice-Hall, Englewood Cliffs, NJ.

[18] Bohner, M., \& Peterson, A. (2001). Dynamic Equations on Time Scales, An Introduction with Applications. Birkhäuser, Boston, Cambridge, MA.

[19] Bohner, M., \& Peterson, A. (2003). Advances in Dynamic Equations on Time Scales. Birkhäuser, Boston, Cambridge, MA.

[20] Denk, A., \& Topal, S. G. (2014). Existence of positive solutions for the second order semipositone m-point boundary value problem. Differential Equations and Dynamical Systems, 22, (3), 265-280.

(C) 2020 by the authors; licensee PSRP, Lahore, Pakistan. This article is an open access article distributed under the terms and conditions of the Creative Commons Attribution (CC-BY) license (http://creativecommons.org/licenses/by/4.0/). 